\title{
Low-Pressure Tactic: A Novel Intrahepatic Shunt Improves Outcomes in Experimental Small-for-Size Syndrome
}

\author{
Toru Goto $^{1,2} \cdot$ Markus Selzner $^{1}$
}

Published online: 18 June 2020

(c) Springer Science+Business Media, LLC, part of Springer Nature 2020

Due to the rapidly growing demand for organ donations, the global severe donor shortage remains a major impediment to liver transplantation. To address this imbalance, living donor liver transplantation has been instituted as an alternative to deceased donor transplantation. Although living donors increase the number of available organs, small-forsize syndrome (SFSS) is a key adverse event that occurs after liver transplantation with small-size grafts, especially for partial liver grafts from living donors. SFSS, defined as dysfunction of a smaller liver graft within 1-2 weeks posttransplantation in the absence of other identifiable causes, is characterized by evidence of hepatic decompensation such as ascites, coagulopathy, cholestasis, and hepatic encephalopathy [1]. SFSS is considered when graft volume/standard liver volume is $<40-50 \%$ or the graft/recipient weight ratio (GRWR) is $<0.8-1.0 \%[2,3]$. Although many factors contribute to the SFSS including donor and recipient status, the primary mechanism is considered to be excessive portal vein flow to the small graft. Portal venous hyperperfusion triggers sinusoidal shear stress, endothelial activation, and nutrient excess that are all thought to directly damage hepatocytes, $[4,5]$ and to induce arterial vasoconstriction, sinusoidal congestion, and hemorrhage [6].

Controlling portal venous pressure is key to attenuating graft damage in the SFSS [7]. To reduce portal flow, splenectomy, splenic artery ligation (SAL), and portocaval shunt have been developed. Although concurrent splenectomy during transplantation improved graft survival by reducing the portal pressure and increasing the vascular compliance of the graft [8], it also led to adverse events such as portal

Markus Selzner

Markus.Selzner@uhn.ca

1 Multi-Organ Transplant Program, Department of Surgery, Toronto General Hospital, Toronto, Canada

2 Division of Hepato-Biliary-Pancreatic Surgery and Transplantation, Department of Surgery, Kyoto University Graduate School of Medicine, Kyoto, Japan venous thrombosis (PVT), pancreatic fistula, and postoperative hemorrhage [9]. SAL, an alternative to splenectomy, exhibited a lower incidence of PVT than splenectomy: SAL $0 \%(n=10)$ versus concurrent splenectomy 3\% $(n=284)$, respectively [10]. Although portocaval shunting reduced portal flow and improved graft survival, it may divert portal flow excessively, leading to the "steal" phenomenon [11]. Furthermore, the need for and timing for closing the shunt are controversial.

In this issue of Digestive Diseases and Sciences, Feng et al. described a novel technique of portosystemic shunt placement during liver transplantation in a rodent SFSS model [12]. They inserted a porous conical tube that had numerous round holes from the portal vein to the suprahepatic vena cava on the "back table," which enabled blood to diffuse to the liver tissue and back into the tube. This novel shunt that the authors termed a trans-portal vein intrahepatic portosystemic shunt (TPIPSS) demonstrated significant prolonged liver filling time from reperfusion, lower portal vein pressure, and improved liver function while preventing hepatocellular injury, enabling a significantly higher survival period in the TPIPSS group. They propose that TPIPSS with absorbable materials, which will disappear after liver function has recovered, might be appropriate for future clinical applications.

TPIPSS is a novel technique that is appealing due to its ease of use in being completed on the "back table" (the secondary table in the operating room). Moreover, it does not prolong the operation time for controlling portal pressure after reperfusion when compared with other portal flow controlling methods. Nevertheless, this maneuver might have several possible limitations. First, the risk of intra-operative vascular and biliary complications is unclear. In a rat model, although the authors only reported one abdominal hemorrhage in each group without any other surgical complication, the possibility of injury to the hepatic artery and hepatic veins, as well as biliary laceration or fistula, needs to be considered. Moreover, since liver regeneration is dependent 
on portal venous flow, the impact of the shunt on hepatocyte proliferation needs to be determined. Furthermore, TPIPSS might increase the risk of hepatic encephalopathy. The TPIPSS group had a higher rate of encephalopathy, which reflects well-established concerns in portosystemic shunt patients. In this method, it is challenging to control the shunt flow after insertion of the tube. Finally, the cumulative survival of 28 days in the TPIPSS group $(60 \%)$, which is significantly superior to the control group, but still below the range that would be generally acceptable for living donor liver transplantation, indicates that additional interventions to control the pathophysiology of SFSS might be needed.

Treatment strategies for SFSS are focused on establishing a balance between diversion of portal flow and liver regeneration. While a number of surgical interventions are currently available, no ideal strategy has been established. The current study presents a novel approach that offers interesting advantages in comparison with more established treatment options. Further studies about the safety, efficacy, and feasibility of TPIPSS will determine whether this strategy can transition from translational research into clinical practice.

\section{Compliance with Ethical Standards}

Conflict of interest The authors of this manuscript have no conflicts of interest to disclose as described by Digestive Diseases and Sciences.

\section{References}

1. Dahm F, Georgiev P, Clavien PA. Small-for-size syndrome after partial liver transplantation: definition, mechanisms of disease and clinical implications. Am J Transpl. 2005;5:2605-2610.
2. Kiuchi T, Kasahara M, Uryuhara K, et al. Impact of graft size mismatching on graft prognosis in liver transplantation from living donors. Transplantation. 1999;67:321-327.

3. Etesami K, Genyk Y. The increasingly limited basis for portal venous pressure modulation in living donor liver transplantation. Liver Transpl. 2018;24:1506-1507.

4. Panis Y, McMullan DM, Emond JC. Progressive necrosis after hepatectomy and the pathophysiology of liver failure after massive resection. Surgery. 1997;121:142-149.

5. Cantre D, Schuett H, Hildebrandt A, et al. Nitric oxide reduces organ injury and enhances regeneration of reduced-size livers by increasing hepatic arterial flow. Br J Surg. 2008;95:785-792.

6. Lautt WW, Legare DJ, Ezzat WR. Quantitation of the hepatic arterial buffer response to graded changes in portal blood flow. Gastroenterology. 1990;98:1024-1028.

7. Ogura Y, Hori T, El Moghazy WM, et al. Portal pressure $<15 \mathrm{~mm}$ $\mathrm{Hg}$ is a key for successful adult living donor liver transplantation utilizing smaller grafts than before. Liver Transpl. 2010;16:718-728.

8. Wang H, Ikegami T, Harada N, et al. Optimal changes in portal hemodynamics induced by splenectomy during living donor liver transplantation. Surg Today. 2015;45:979-985.

9. Ito K, Akamatsu N, Ichida A, et al. Splenectomy is not indicated in living donor liver transplantation. Liver Transpl. 2016;22:1526-1535.

10. Yoshizumi T, Mori M. Portal flow modulation in living donor liver transplantation: review with a focus on splenectomy. Surg Today. 2020;50:21-29.

11. Troisi R, Ricciardi S, Smeets P, et al. Effects of hemi-portocaval shunts for inflow modulation on the outcome of small-forsize grafts in living donor liver transplantation. Am J Transpl. 2005;5:1397-1404.

12. Feng Y, Han Z, Gu B, et al. A novel method for the prevention and treatment of small-for-size syndrome in liver transplantation. Dig Dis Sci. (Epub ahead of print). https://doi.org/10.1007/s1062 0-020-06055-2.

Publisher's Note Springer Nature remains neutral with regard to jurisdictional claims in published maps and institutional affiliations. 